DOI: https://doi.org/10.11144/Javeriana.umed61-4.semi

\title{
Semiología de las alucinaciones en tumores del sistema nervioso central
}

Semiology of Hallucinations in Central Nervous System Neoplasms

Recibido: 28/11/2019 | Aceptado: 28/04/2020

Juan Carlos Acevedo-González

Médico neurocirujano. Especialista en Neurocirugía

Funcional, Estereotaxia, Manejo de Dolor y de

Espasticidad. Profesor del Departamento de

Neurociencias de la Facultad de Medicina de la

Pontificia Universidad Javeriana, Bogotá, Colombia

María Lucía Salamanca-Ternera ${ }^{a}$

Médica, Pontificia Universidad Javeriana, Bogotá,

Colombia

a Autora de correspondencia: msalamancat@javeriana.edu.co

Cómo citar: Acevedo-González JC, SalamancaTernera ML. Semiología de las alucinaciones en tumores del sistema nervioso central. Univ. Med. 2020;61(4). https://doi.org/10.11144/Javeriana.umed 61-4.semi

\section{RESUMEN}

Las alucinaciones son un fenómeno complejo presente en diferentes entidades médicas psiquiátricas y no psiquiátricas. Una de las condiciones que presentan alucinaciones como parte de sus síntomas es el tumor del sistema nervioso central. Estos corresponden al $2 \%$ entre todas las neoplasias y tienen un componente importante de morbimotalidad, en parte por los efectos de los síntomas en la calidad de vida del individuo. La adecuada aproximación diagnóstica e identificación de la etiología de este síntoma determinará su pronóstico y alternativas de manejo que varían según la enfermedad que los produzca; en el caso de los tumores del sistema nervioso central habrá alguna posibilidad de tratamiento definitivo con intensiones curativas. Este artículo busca documentar características semiológicas de las alucinaciones que le permitan al médico en la práctica clínica evaluarlas con mayor claridad en el momento de la aproximación diagnóstica, en especial las características de su presentación en el contexto del cáncer cerebral y diferenciarlo de otras posibles causas.

Palabras clave

alucinaciones; neoplasias encefálicas; errores diagnósticos; cerebro; técnicas de diagnóstico neurológico.

\section{ABSTRACT}

Hallucinations are a complex phenomenon present in different psychiatric and non-psychiatric medical entities. One of the conditions that present hallucinations as part of its symptoms is the central nervous system tumor. Tumors in the central nervous system (CNS) correspond to 2 $\%$ of all malignancies and have an important component of morbidity and mortality, in part, due to the effects that the symptoms have on the quality of life of the person. The proper diagnostic approach and identification of the etiology of this symptom will determine the prognosis and management alternatives that will vary, depending on the disease that produces it, in the case of central nervous system tumors, some possibility of definitive treatment with curative intentions. The objective of this text is to present semiological characteristics of hallucinations that allow the 
doctor in practice to evaluate the symptom more clearly at the time of the diagnostic approach, specially the characteristics of its presentation in the context of brain cancer and differentiate it from other possible causes.

Keywords

hallucinations; brain neoplasms; diagnostic errors; cerebrum; diagnostic techniques; neurological.

\section{Introducción}

Según la última actualización del Manual diagnóstico y estadístico de los trastornos mentales (DSM-5, por sus siglas en inglés), una alucinación es una percepción sensorial falsa en ausencia de un estímulo sensorial relacionado (1). Las alucinaciones son un fenómeno complejo activo en diferentes entidades médicas psiquiátricas y no psiquiátricas (2-4). Al hablar de estas, es frecuente remitirse a la psicosis, caracterizada por desorganización del pensamiento, afecto o comportamiento (5-7); sin embargo, es importante eliminar esta premisa, al evaluarlas en la práctica clínica, ya que hay situaciones en las cuales surgen y no serán en el contexto de la psicosis $(2,8,9)$. Las alucinaciones a las que nos referimos en este artículo, para considerarse como tal, deben presentarse cuando un individuo está despierto y en completo estado de alertamiento. Esto significa que no se incluyen las alucinaciones que aparecen durante el síndrome confusional agudo, también conocido como delirium, o en los trastornos del sueño $(3,7,10,11)$.

Una de las condiciones que presentan las alucinaciones como parte de sus síntomas son los tumores del sistema nervioso central (SNC) $(12,13)$, los cuales corresponden al $2 \%$ entre todas las neoplasias y tienen un componente importante de morbimortalidad $(14,15)$, en parte por los efectos que los síntomas generan en la calidad de vida del individuo (16-18). Cabe anotar que aun cuando la mayoría de los pacientes psiquiátricos no cursan con neoplasias del SNC, sí es frecuente que los tumores cerebrales surjan con alguna alteración mental en algún momento de la enfermedad (19). Esto ha generado la necesidad de realizar estimaciones de la cantidad de pacientes en centros psiquiátricos que tendrían otro tipo de enfermedad médica no psiquiátrica no diagnosticada, de lo que se han obtenido indicadores que han evolucionado desde cifras tan altas como uno de cada 30 pacientes (cuando no se contaba con los avances imagenológicos) hasta uno de cada 200 pacientes (16). En cuanto a los pacientes con neoplasias del SNC, se ha identificado que la presencia de la sintomatología de carácter psiquiátrico disminuye de manera importante su calidad de vida, siendo los síntomas depresivos en pacientes con tumor cerebral el predictor más importante (16).

Muchos trastornos pueden inducir psicosis, y varios de ellos son relativamente comunes (4). Sin embargo, son pocos los estudios y no es posible saber con certeza qué tan frecuente se ven estas alteraciones (16). Por lo mismo, no es raro que a estos pacientes se les diagnostique de manera errónea esquizofrenia o alguna otra psicosis (20). En consecuencia, no hay datos confiables para determinar una incidencia y prevalencia de alucinaciones secundarias a tumores del SNC (16). Por eso, cobra gran importancia el tema que se busca tratar a lo largo de este escrito.

Teniendo en cuenta lo descrito, se ha reconocido la importancia de cambiar los patrones de abordaje de estos pacientes y se ha identificado la necesidad de aclarar conceptos para evaluar profundamente al paciente con síntomas psiquiátricos; en este caso, con alucinaciones, y evitar pasar por alto una alteración médica no psiquiátrica (20). La adecuada aproximación diagnóstica e identificación de la etiología de este síntoma determinará alternativas de manejo que varían según la enfermedad que los produzca. En el caso de los tumores del SNC, habrá alguna posibilidad de tratamiento definitivo con intenciones curativas, cambiando de manera abismal el abordaje terapéutico y pronóstico para el paciente $(15,16,21,22)$.

\section{Metodología}

Para la elaboración de este artículo se revisó la literatura sobre el tema en el megabuscador 
de la Biblioteca de la Pontificia Universidad Javeriana y se incluyeron las siguientes bases de datos: Medline vía PubMed, ScienceDirect, Clinical Key, Dialnet, MedicLatina y Scopus. Los términos $\mathrm{MeSH}$ utilizados en la búsqueda fueron: alucinaciones, psicosis, neoplasias del SNC, tumores del SNC, neuropsiquiatría, psiquiatría orgánica, alucinaciones no psiquiátricas. La búsqueda incluyó artículos en español y en inglés. Adicionalmente, se revisaron libros de texto reconocidos en el estudio de la psiquiatría y la psiquiatría orgánica.

\section{Características generales de las alucinaciones}

Las alucinaciones son casi siempre anormales y pueden afectar cualquiera de los sentidos, y entre estas las más comunes son las auditivas y las visuales. Por lo general, las primeras son características de algunas enfermedades psiquiátricas; mientras que se considera que las alucinaciones no auditivas sugieren alguna condición médica no psiquiátrica $(1,23)$. No debe confundirse la alucinación con la ilusión, que es el fenómeno de una percepción errada a un estímulo real, usualmente momentánea (24). En la tabla 1 se exponen algunos términos importantes para caracterizar las alucinaciones.

\section{Tabla 1}

\section{Clasificación de las alucinaciones}

\begin{tabular}{|l|l|}
\hline $\begin{array}{l}\text { Alucinaciones } \\
\text { auditivas }\end{array}$ & $\begin{array}{l}\text { Falsa percepción del sonido, usualmente voces, pero puede darse } \\
\text { con ruidos y música. Es el tipo de alucinación más común en } \\
\text { enfermedades psiquiátricas primarias. }\end{array}$ \\
\hline $\begin{array}{l}\text { Alucinaciones } \\
\text { visuales simples }\end{array}$ & Destellos de luces, patrones geométricos o colores. \\
\hline $\begin{array}{l}\text { Alucinaciones } \\
\text { visuales complejas }\end{array}$ & $\begin{array}{l}\text { De naturaleza caleidoscópica, escenas completas, con personas y } \\
\text { animales. }\end{array}$ \\
\hline $\begin{array}{l}\text { Alucinaciones } \\
\text { olfatorias }\end{array}$ & $\begin{array}{l}\text { Principalmente relacionadas con olores y hedores; son más } \\
\text { frecuentes secundarias a enfermedades no psiquiátricas, } \\
\text { especialmente del lóbulo temporal o tumores del área olfativa. }\end{array}$ \\
\hline $\begin{array}{l}\text { Alucinaciones } \\
\text { gustativas }\end{array}$ & $\begin{array}{l}\text { Percepción de sabores, por lo general desagradables. Pueden } \\
\text { acompañarse de ideación delirante secundaria. Por ejemplo, "me } \\
\text { envenenan la comida". Son especialmente sugerentes de } \\
\text { enfermedad médica no psiquiátrica o consumo de sustancias. }\end{array}$ \\
\hline $\begin{array}{l}\text { Alucinaciones } \\
\text { táctiles o hápticas }\end{array}$ & $\begin{array}{l}\text { Sensaciones cutáneas de animales pequeños corriendo bajo la piel, } \\
\text { formicación. El paciente puede presentar lesiones de rascado } \\
\text { producidas al buscar los pequeños insectos. } \\
\text { Comunes en intoxicación por cocaína, abstinencia de alcohol y en } \\
\text { patologías de base no psiquiátrica. }\end{array}$ \\
\hline $\begin{array}{l}\text { Alucinación } \\
\text { somática o } \\
\text { cinestésica }\end{array}$ & $\begin{array}{l}\text { Percepción de una experiencia fisica relacionada con el propio } \\
\text { funtasma. }\end{array}$ \\
\hline $\begin{array}{l}\text { Alucindrome de Cotard o sindrome de miembro } \\
\text { extrañas }\end{array}$ & $\begin{array}{l}\text { Material alucinatorio distorsionado, como las alucinaciones } \\
\text { lilliputianas, donde los objetos y personas son diminutas. }\end{array}$ \\
\hline
\end{tabular}

En el momento de evaluar a un paciente con alucinaciones es importante tener en cuenta que su contenido varía según el contexto cultural (25) y la personalidad de base del paciente (24).
Por ello, es precisa una aproximación integral e individual, para determinar su origen (26) (4).

\section{Alucinaciones en enfermedades médicas} psiquiátricas y no psiquiátricas

Cuando se le hace la anamnesis a un paciente con alucinaciones debe indagarse por las características propias de la alucinación, el tiempo de evolución y otros síntomas asociados, así como indagar por aspectos culturales del paciente $(4,12)$. En la tabla 2 se exponen algunas recomendaciones que son de gran ayuda para guiar el diagnóstico y diferenciar una alucinación primaria de una secundaria (24).

\section{Tabla 2}

Aspectos que se deben evaluar en un paciente con alucinaciones

\begin{tabular}{|c|c|c|}
\hline Aspectos & $\begin{array}{c}\text { Enfermedad médica no } \\
\text { psiquiátrica }\end{array}$ & Psicosis \\
\hline $\begin{array}{l}\text { ¿Qué siente el paciente cuando } \\
\text { suceden estas percepciones? }\end{array}$ & $\begin{array}{l}\text { Hay algo de sensación de } \\
\text { irrealidad en el paciente. }\end{array}$ & Suelen ser muy vívidas. \\
\hline ¿En qué momento suceden? & $\begin{array}{l}\text { Tiene una aparición cíclica, a } \\
\text { un momento del día } \\
\text { determinado, p. ej., sindrome } \\
\text { crepuscular. }\end{array}$ & Suelen ser impredecibles. \\
\hline $\begin{array}{l}\text { ¿Tienen un patrón relacionado } \\
\text { con alguna actividad? }\end{array}$ & \begin{tabular}{|l|} 
Pueden relacionarse con \\
alguna actividad en concreto.
\end{tabular} & $\begin{array}{l}\text { Suelen aparecer en cualquier } \\
\text { momento. }\end{array}$ \\
\hline $\begin{array}{l}\text { Patrón de frecuencia iSon } \\
\text { diarias o esporádicas? }\end{array}$ & $\begin{array}{l}\text { Pueden ser o diarias o } \\
\text { esporádicas. }\end{array}$ & \begin{tabular}{|l} 
Suele ser constantes. \\
\end{tabular} \\
\hline $\begin{array}{l}\text { Ubicación de la alucinación } \\
\text { dentro de su cabeza o fuera de } \\
\text { esta }\end{array}$ & $\begin{array}{l}\text { Usualmente el paciente ubica } \\
\text { las alucinaciones dentro de } \\
\text { la cabeza. }\end{array}$ & Puede ser cualquiera de los dos. \\
\hline $\begin{array}{l}\text { ¿Qué tan nítidas son? (P. ej., } \\
\text { en caso de las alucinaciones } \\
\text { auditivas si son sonidos, } \\
\text { palabras, órdenes o } \\
\text { conversaciones). }\end{array}$ & $\begin{array}{l}\text { Tienden a ser alucinaciones } \\
\text { simples, poco nítidas. }\end{array}$ & $\begin{array}{l}\text { Puede haber alucinaciones } \\
\text { complejas y muy vívidas. }\end{array}$ \\
\hline $\begin{array}{l}\text { ¿Son egosintónicas o } \\
\text { egodistónicas? (El paciente se } \\
\text { incomoda o no con la } \\
\text { presencia de estas } \\
\text { percepciones). }\end{array}$ & $\begin{array}{l}\text { El paciente tiene algo de } \\
\text { introspección y le incomoda } \\
\text { en algún porcentaje la } \\
\text { presencia de las } \\
\text { alucinaciones. } \\
\text { Egodistónicas. } \\
\end{array}$ & $\begin{array}{l}\text { El paciente acepta las } \\
\text { alucinaciones como parte de la } \\
\text { realidad. No tiene } \\
\text { introspección. Son eg- } \\
\text { sintónicas. }\end{array}$ \\
\hline $\begin{array}{l}\text { ¿Hay asociación con alguna } \\
\text { alteración cognitiva? }\end{array}$ & $\begin{array}{l}\text { Puede haber alteración del } \\
\text { estado de conciencia o } \\
\text { atención. }\end{array}$ & $\begin{array}{l}\text { Atención y estado de conciencia } \\
\text { conservado. Posiblemente algo } \\
\text { de seudoaprosexia. }\end{array}$ \\
\hline Percepción del propio cuerpo. & Asociación con agnosias. & $\begin{array}{l}\text { No hay una alteración de la } \\
\text { percepción del propio cuerpo, } \\
\text { sino del entorno. }\end{array}$ \\
\hline Momento de inicio. & $\begin{array}{l}\text { Inician poco después de una } \\
\text { alteración física. }\end{array}$ & $\begin{array}{l}\text { No se encuentra asociación con } \\
\text { alteraciones fisicas. }\end{array}$ \\
\hline $\begin{array}{l}\text { Remisión tras el tratamiento } \\
\text { del problema fisico. }\end{array}$ & $\begin{array}{l}\text { Remiten en gran proporción } \\
\text { los sintomas. }\end{array}$ & No remiten los sintomas. \\
\hline $\begin{array}{l}\text { Proporcionalidad de los } \\
\text { sintomas. }\end{array}$ & \begin{tabular}{|l|} 
Al tiempo que el trastorno \\
fisico se agrava, también lo \\
hacen los sintomas mentales, \\
conductuales y emocionales. \\
\end{tabular} & $\begin{array}{l}\text { No es proporcional. Mejora o } \\
\text { empeora sin asociación con un } \\
\text { trastorno fisico. }\end{array}$ \\
\hline $\begin{array}{l}\text { Presencia de conexión } \\
\text { fisiológica conocida entre la } \\
\text { condición fisica y el síntoma } \\
\text { en cuestión. }\end{array}$ & $\begin{array}{l}\text { El trastorno físico debe } \\
\text { reconocerse como capaz de } \\
\text { inducir el sintoma. }\end{array}$ & $\begin{array}{l}\text { Los síntomas no se explican } \\
\text { fisiopatológicamente con una } \\
\text { lesión. }\end{array}$ \\
\hline
\end{tabular}

En muchas ocasiones, los síntomas no van a ser claros ni fáciles de evaluar; será complejo determinar solo con la semiología si la afección mental se debe a una enfermedad médica psiquiátrica o a una no psiquiátrica. Lo expuesto en la tabla 2 no es un absoluto; es una guía general. La presentación de los síntomas puede 
ser muy variable y el paciente debe evaluarse de manera individualizada (12).

Las alucinaciones de origen no psiquiátrico usualmente son visuales, olfatorias y táctiles, y rara vez son auditivas; mientras que las alucinaciones típicas de psicosis como en esquizofrenia son auditivas $(4,27)$. Estas últimas se describen como voces amenazantes, obscenas, acusatorias, conversaciones entre dos o más voces, o simplemente una voz que narra la vida del paciente. Las alucinaciones visuales también se ven frecuentemente en el contexto de las psicosis $(24,28,29)$.

Es importante anotar que las alucinaciones secundarias a procesos no psiquiátricos pueden acompañarse de ideas delirantes pobremente elaboradas con cierto componente de duda, a diferencia de las primarias, que pueden llegar a tener incluso algo de confabulación (falsos recuerdos de los que el paciente no es consciente, con ferviente y genuina creencia en la veracidad del recuerdo) $(30,31)$. El tipo de idea delirante, al igual que parte del contenido de las alucinaciones, tiene que ver también con la personalidad premórbida del paciente y de su contexto. Si se tiene a un paciente con rasgos esquizotípicos de la personalidad, es más probable que se presente con fuertes ideas delirantes, relacionadas con el pensamiento de tipo mágico que ha tenido siempre por su personalidad de base (31).

En el caso del cáncer, el tumor en sí podría actuar como elemento precipitante para la aparición de enfermedades mentales en personas susceptibles, es decir, un paciente puede desarrollar alguna enfermedad psiquiátrica (esquizofrenia, depresión, etc.) adicional a la aparición del tumor (12), en parte desencadenado por el síndrome cerebral agudo. Lo característico de este escenario es que la sintomatología no se resuelve una vez tratado el proceso neoplásico (16).
Mecanismos fisiopatológicos de los síntomas en los tumores de sistema nervioso central

Los tumores del SNC tienen una particularidad: independientemente de si se clasifican en benignos o malignos, su localización puede determinar la magnitud de la sintomatología, incluso llevándola a ser letal (14). Los más frecuentes son las metástasis, que representan el $50 \%$ de todas las neoplasias intracraneales, y los principales tumores metastásicos a cerebro son los tumores de pulmón, seno, digestivos y melanomas. Otros no tan frecuentes son colorrectal y renal. En cuanto a los tumores primarios, los gliomas son los más frecuentes, seguidos por los meningiomas, que entre los dos llegan a ser más del 50 \% (14,19,32,33).

Hay variedad de síntomas en los tumores del SNC (34) que abarcan desde focalizaciones (alteraciones manifiestas de lesión en un lugar puntual del cerebro) hasta signos neurológicos blandos (35) (alteraciones de funciones que abarcan diferentes partes del cerebro, como las alucinaciones). La localización del tumor puede representar un riesgo de letalidad, en especial si tienen afectación del tallo cerebral $(18,36)$.

Todos los cambios secundarios a un tumor en el cerebro son capaces de producir un cuadro clínico conocido como síndrome cerebral agudo, definido como aquellas manifestaciones - usualmente de inicio súbito- en un paciente sano, de una alteración bioquímica, eléctrica o mecánica del cerebro. La mayoría son de carácter reversible, si se corrige la causa subyacente. Dentro de este síndrome se pueden presentar alucinaciones y es posible que el paciente persista con ideas delirantes en lo que respecta a las experiencias alucinatorias que tuvo durante el síndrome (37).

Diferentes mecanismos generan los síntomas en los tumores del SNC (21): el efecto de masa, el edema peritumoral y la localización en sí del tumor. Así mismo, la velocidad de crecimiento del tumor va a tener un papel importante en las manifestaciones clínicas (38). Teniendo en cuenta lo descrito, las características histológicas del tumor serían capaces de predecir, en cierta 
medida, el pronóstico del cuadro en términos de su velocidad de crecimiento y sus lugares frecuentes de aparición que, como se muestra adelante, se presentará en alucinaciones con características específicas.

Los síntomas generalizados se relacionan con el crecimiento y compresión secundaria, (cefalea, náuseas, papiledema y crisis convulsivas); mientras que los síntomas focales se relacionan más con la localización y extensión del tumor $(16,21)$. En cuanto a los síntomas determinados por la velocidad del crecimiento celular en el área mental, los tumores de crecimiento lento tienden a producir cambios en la personalidad y permiten la manifestación de alteraciones psíquicas a las que el paciente era previamente susceptible; en tanto que los tumores de crecimiento más rápido se relacionan más con alteraciones de carácter cognitivo $(16,19)$.

Dentro de las neoplasias del SNC, las metástasis, especialmente cuando son múltiples, tienen mayor prevalencia de síntomas psiquiátricos que cualquiera de los tumores primarios (33). Hasta el momento no se ha identificado un patrón histológico con una mayor probabilidad de presentar algún síntoma psiquiátrico en específico. Sin embargo, de las primarias, se han registrado los gliomas como los de más alta incidencia en alteraciones mentales, llegándose a calcular que un 70\%-80\% de los pacientes con glioblastomas evidencian síntomas psiquiátricos, y un $25 \%-35 \%$, de los pacientes cursan con astrocitomas de bajo grado (16). Se ha descrito que las secuelas psiquiátricas de los meningiomas están en importante relación con la extensión de edema peritumoral, más que con el tamaño del tumor en sí. Esto puede atribuirse a la disfunción de las vías cerebrales por el edema $(16,21)$.

Las alteraciones de la formación reticular del tallo cerebral, su proyección rostral a la corteza, la compresión directa del tejido cerebral, la circulación inadecuada o alteraciones del flujo de líquido cefalorraquídeo en el contexto del cáncer en el cerebro ha resultado en un aumento de le presión intracraneana, que constituye otra de las causas de los síntomas neuropsiquiátricos $(21,39)$.
Así mismo, los cambios en el encéfalo producidos por los tumores son capaces de originar focos epileptógenos (21). Una de las manifestaciones clínicas más importantes de las neoplasias en el cerebro son las crisis convulsivas, algunas de las cuales se manifiestan como alucinaciones. En el contexto, estas pueden ser de cualquier tipo, tener una clara asociación con algún foco pero también se pueden dar sin evidencia de dicha actividad. La naturaleza de la alucinación dependerá de la localización del tumor. En general, relacionadas con las lesiones del lóbulo occipital que cursan con alteraciones visuales y del lóbulo temporal con crisis sensitivas (gustativas, auditivas, olfatorias, entre otras) $(16,40)$.

Las crisis iniciadas en la corteza visual primaria se asocian con alucinaciones visuales simples; mientras que alucinaciones auditivas con percepción de sonidos de zumbido y siseo se han visto en focos de la corteza auditiva primaria en medio del giro superior temporal o circunvoluciones transversas de Heschl. En cuanto a las experiencias de tipo olfatorio y gustativo (a quemado o metálico), se asocian con focos del sistema límbico $(24,41)$.

Alucinaciones y otros síntomas psiquiátricos en el contexto de un tumor del sistema nervioso central según localización

Las manifestaciones psiquiátricas son de poca utilidad para sugerir la localización exacta y el tipo de tumor. Es complejo diferenciar las alteraciones mentales producidas directamente por el tumor a las secundarias a efectos de masa o a efectos generales del aumento de la presión intracraneana.

La localización de los tumores se manifiesta de diferentes maneras en el paciente, por ejemplo, las alteraciones mentales en general son más frecuentes en tumores del lóbulo frontal y temporal que en tumores parietales y occipitales; mientras que lesiones del lóbulo temporal y occipital presentan alucinaciones en mayor proporción. Las lesiones que involucran el lóbulo temporal y el lóbulo parietal, 
especialmente del hemisferio derecho, se asocian con ideación delirante. Los pacientes que evidencian disminución sensorial (auditiva o visual), secundaria $\mathrm{o}$ no a algún proceso neoplásico, son propensos a alucinaciones y experiencias delirantes como mecanismo de autoestimulación (24).

\section{Lóbulo frontal}

Los tumores de esta localización son proclives a alteraciones mentales, caracterizadas por ser síntomas de etapas tempranas de la enfermedad, cambios de personalidad, conductas inusuales, desinhibiciones y pensamientos intrusivos en algunos pacientes (42). En cuanto a las alucinaciones, los tumores circunscritos al lóbulo frontal pueden producir ocasionalmente alucinaciones visuales, auditivas (43) e incluso gustativas. Se ha descrito que los tumores de base de cráneo casi no tienen manifestaciones psiquiátricas; mientras que en lesiones de la convexidad se ve depresión y psicosis. Los tumores que afectan ambos hemisferios o se ubican en la línea media son más sintomáticos (16).

\section{Lóbulo temporal}

Los tumores en esta área producen la mayor cantidad de sintomatología psiquiátrica. En parte por ser propensa a la actividad epiléptica (16). Están asociados con alucinaciones visuales complejas (44) y alucinaciones auditivas, muy similares a las que se presentan en esquizofrenia. Es común que los pacientes sientan muy vívidas las alucinaciones y poco tiempo después tengan introspección y conciencia de su naturaleza irracional. Las alucinaciones asociadas con hemianopsia son particularmente características de lesiones del lóbulo temporal, así como alucinaciones gustativas y olfatorias que se relacionan más con las lesiones de la región ulcinada. En imágenes diagnósticas de pacientes con alucinaciones auditivas se ha identificado un aumento del flujo sanguíneo en el área de Broca y disminución del flujo sanguíneo en las áreas motoras suplementarias y corteza temporal izquierda (42).

Hay unas alucinaciones especiales que surgen de lesiones del lóbulo temporal. Estas son las relacionadas con la percepción del cuerpo fuera de sí, que involucran tres tipos: autoscópicas, heautoscópicas y experiencias extracorporales. Las primeras hacen referencia a la percepción visual del propio cuerpo fuera de sí mismo, reconociéndose desde el cuerpo "real" (p. ej., veo un cuerpo igual al mío). Se deben principalmente a lesiones occipitotemporales. En las alucinaciones heautoscópicas, que son un estado intermedio entre las alucinaciones autoscópicas y las experiencias extracorporales, la persona no es capaz de reconocer cuál es su cuerpo real, y son más frecuentes en lesiones frontotemporales y región posterior de la ínsula, principalmente del hemisferio izquierdo (42). Por último, las experiencias extracorporales son las que dan una sensación de salida del propio cuerpo, viendo al cuerpo "real" desde fuera (p. ej., veo mi cuerpo en la cama y no estoy dentro de él).

\section{Lóbulo parietal}

Las alucinaciones secundarias a lesiones del lóbulo parietal son predominantemente cinestésicas y táctiles, es decir, relacionadas con sensaciones corporales en ausencia de estímulo real, como en el síndrome de miembro fantasma, representadas en el hemicuerpo contralateral. Se ha descrito también un fenómeno alucinatorio donde persiste la sensación del estímulo táctil tiempo después de que el estímulo se ha retirado (16).

\section{Lóbulo occipital}

Las alteraciones de presentación aguda o de crecimiento rápido en la región occipital se muestran frecuentemente con alteraciones perceptivas importantes, con ilusiones y alucinaciones visuales simples, sobre todo como destellos de luces o figuras. Las alucinaciones 
visuales complejas se dan más en lesiones del lóbulo no dominante (16).

Hay una particularidad con las lesiones que afectan la visión: producen disminución sensorial, como las hemianopsias; en ocasiones crean alucinaciones visuales simples. Cuando hay una lesión bilateral y ceguera cortical (que se distingue de la periférica por la conservación de reflejo pupilar), podría darse la situación, como en el síndrome de Charles Bonnet (24), en el cual pacientes que después de lesiones cerebrales o periféricas pierden alguna función sensorial, como la vista o el oído y, paradójicamente, presentan alucinaciones complejas relacionadas con la función perdida $(45,46)$.

Las lesiones en el lóbulo occipital son poco específicas en su sintomatología, sobre todo en manifestaciones distintas a la vista. La agnosia visoespacial, con dificultades para el reconocimiento de imágenes y percepción tridimencional, ocurre más frecuentemente en lesiones del lóbulo no dominante y puede cursar con morphopsia, condición en la cual los objetos son percibidos de manera distorsionada (16).

\section{Cuerpo calloso}

Se ha descrito la presencia de síntomas floridos de psicosis y, en ocasiones, sintomatología similar a la esquizofrenia en tumores o lesiones del cuerpo calloso. Dependiendo de la localización, hay mayor o menor incidencia de cambios mentales. Los tumores que involucran el rostrum presentan sintomatología psiquiátrica en un $92 \%$, un $89 \%$ en tumores del esplenio y un $57 \%$ en tumores del cuerpo (16).

\section{Tallo cerebral y fosa posterior}

Algunos tumores de esta región se presentan con alucinosis peduncular, que es un fenómeno que se caracteriza por alucinaciones visuales complejas, muy coloridas que combinan las sensaciones visuales-acústicas y visuales-táctiles. Usualmente, son autolimitadas y se resuelven espontáneamente. Pueden darse alucinaciones visuales por compresión de la corteza del lóbulo occipital $(16,47)$.

\section{Aproximación diagnóstica}

Una vez identificado un episodio alucinatorio en una persona previamente sana es indispensable realizarle estudios imagenológicos, sea en un primer episodio psicótico o cuando se tenga sospecha clínica clara de un posible secundarismo. La tomografía axial computarizada es el estudio usual de abordaje inicial; sin embargo, tiene menor sensibilidad y especificidad que la resonancia magnética para evaluar detalladamente las características del tumor. Se usa la tomografía cuando el paciente tiene contraindicación para una resonancia, cuando hay urgencia de tomar la imagen o cuando hay sospecha de lesiones que infiltran hueso. La resonancia magnética nuclear con gadolinio es el estudio de elección, por el detalle de la imagen y por la captación del contraste, dados ciertos tumores que ayudan a una mejor aproximación a la posible etiología $(33,48)$.

Una vez se tenga clara la causa etiológica de la sintomatología, es necesario que el médico realice adecuadamente la clasificación diagnóstica. En una enfermedad médica no psiquiátrica se debe registrar primero la afección médica de base, y dependiendo de los síntomas predominantes se agrega al diagnóstico "con ideas delirantes" o "con alucinaciones". Puede especificarse la gravedad si se considera necesario (1), por ejemplo, trastorno psicótico debido a carcinoma metastásico, con alucinaciones (F06.0) $(49,50)$.

\section{Diagnósticos diferenciales}

Otras afecciones médicas no psiquiátricas que pueden causar psicosis incluyen epilepsia del lóbulo temporal, trastornos endocrinos (como la enfermedad tiroidea y la suprarrenal), estados de deficiencia vitamínica, sífilis del SNC, esclerosis múltiple, lupus eritematoso sistémico, enfermedad de Wilson, uso de sustancias psicoactivas, secundario a medicamentos, abstinencia alcohólica, trastornos del sueño y 
traumatismo craneoencefálico (29,51-53). En los adultos mayores, las alucinaciones e ilusiones pueden darse por fenómenos transitorios secundarios a una disminución sensorial (p. ej., delírium o síndrome de Charles-Bonnet) $(4,54)$.

\section{Conclusiones}

Los tumores del SNC pueden manifestar una gran variedad de síntomas, desde muy inespecíficos y generalizados, como la cefalea, hasta muy propios de una localización precisa en el cerebro, como alteraciones en el examen neurológico. Los grandes determinantes de las implicaciones del tumor son la tasa de crecimiento celular y la localización en el cerebro; de esto depende que un cáncer sea de carácter curativo o sea potencialmente letal.

Dentro de las manifestaciones clínicas, los tumores del SNC presentarán con frecuencia sintomatología psiquiátrica, que muchas veces podrá afectar de manera importante la vida de estos pacientes. Las alucinaciones hacen parte de este grupo, las cuales dificultan el diagnóstico, al no ser estudiadas como posible manifestación de un proceso secundario a una enfermedad no psiquiátrica del SNC y ser atribuidas a patologías psiquiátricas primarias. Las alteraciones de tipo alucinatorio están determinadas en parte por el sitio de localización tumoral y en parte por la extensión del edema peritumoral, ya que este último altera el adecuado funcionamiento de las vías neuronales. Las alucinaciones más sugestivas de procesos orgánicos son las que involucran los sentidos diferentes al oído; estas suelen evidenciarse en los lóbulos frontal, temporal y occipital, usualmente con ideación delirante pobremente estructurada y posteriormente con cierta capacidad de introspección y reconocimiento de la irrealidad de las alucinaciones.

En el contexto de los tumores cerebrales, hay varios mecanismos que explican la fisiopatología de las alucinaciones: por afectación directa de las vías sensoriales, por mal funcionamiento de las vías cerebrales, por edema peritumoral, por aumento de la presión intracraneana, por la presencia de focos epileptógenos, por disminución sensorial asociados a fenómenos de autoestimulación por medio de las alucinaciones, entre otros. Por lo anterior, es de gran importancia una buena aproximación diagnóstica en estos pacientes, con un examen neurológico completo que evalúe puntos clave de las alucinaciones y con imágenes diagnósticas que ayuden a enfocar al paciente y, de esta manera, evitar que se difiera y no se trate oportunamente una condición médica que lo amerita.

\section{Bibliografía}

1. Morrison J. DSM-5: guía para el diagnóstico clínico. México: Manual Moderno; 2014.

2. Freudenreich $\mathrm{O}$, Brown $\mathrm{H}$ y Holt D. Psicosis y esquizofrenia. En: Massachusetts General Hospital: tratado de psiquiatría clínica. 2. a ed. Barcelona: Elsevier; 2017.

3. Huff, JS. Confusion. Rosen's emergency medicine: concepts and clinical practice. $9^{\text {th }}$ ed. New York: Elsevier; 2018.

4. Waters F, Fernyhough C. Hallucinations: a systematic review of points of similarity and difference across diagnostic classes. Schizophr Bull. 2017;43(1):32-43. https://doi.org/ 10.1093/schbul/sbw132

5. Cremens MC. Psiquiatría geriátrica. En: Massachusetts General Hospital: tratado de psiquiatría clínica. 2. a ed. Barcelona: Elsevier; 2017.

6. Téllez JM, Villena A, Morena S, Martínez M, Ballesteros M. El paciente delirante. Barcelona: Elsevier; 2018.

7. Luttrull MD, Boulter DJ, Kirsch CFE, et al. ACR Appropriateness Criteria acute mental status change, delirium, and new onset psychosis. J Am Coll Radiol. 2019;46(5S):S26S37. https://doi.org/.1016/j.jacr.2019.0 2.024 
8. Lo YL, Hameed S, Rumpel H, Chan LL. Auditory hallucinations and migraine of possible brainstem origin. J Headache Pain. 2011;12(5):573-5. htt ps://doi.org/.1007/s10194-011-0355-z

9. Meppelink AM. Imaging in visual hallucinations. En: The neuroscience of visual hallucinations. Nueva York: John Wiley \& Sons; 2015. p. 151-6.

10. Castellano M, Sánchez C, Gisbert JA. Trastornos mentales orgánicos. En: Gisbert Calabuig: medicina legal y toxicológica. Barcelona: Elsevier; 2019p. 1202-20.

11. Sherwood SJ. A web survey of the content, sensory modalities, and interpretation of hypnagogic and hypnopompic experiences. J Parapsychol. 2012;76(1):27-55.

12. Carota A, Bogousslavsky J. Neurology versus psychiatry? Hallucinations, delusions, and confabulations. Front Neurol Neurosci. 2019;44:127-40. https://doi.org/.1159/ 000494960

13. Kosty JA, Mejia-Munne J, Dossani R, Savardekar A, Guthikonda B. Jacques Jean Lhermitte and the syndrome of peduncular hallucinosis. Neurosurg Focus. 2019;47(3):E9. https ://doi.org/.3171/2019.6.FOCUS19342.

14. Pardo C, Cendales R. Incidencia, mortalidad y prevalencia de cáncer en Colombia. Bogotá: Instituto Nacional de Cancerología; 2015.

15. Gould J. Breaking down the epidemiology of brain cancer. Nature.2018;561:S40-1.

16. David D, Fleminger S, Kopelman M, Lovestone S, Mellers J, editores. Lishman's organic psychiatry: a textbook of neuropsychiatry. 4th ed. Nueva York: Wiley; 2011.

17. Boele FW, Rooney AG, Grant R, Klein M. Psychiatric symptoms in glioma patients: from diagnosis to management. Neuropsychiatr Dis Treat. 2015;11:1413-20.

18. Schiff D, Lee EQ, Nayak L, Norden AD, Reardon DA, Wen PY. Medical management of brain tumors and the sequelae of treatment. NeuroOncology. 2015;17:488-504.

19. Gómez-Utrero E, Navarro F, López JL, Álvarez-Mon Soto M. Complicaciones neurológicas del cáncer. Medicine: Programa de Formación Médica Continuada Acreditado. 2017;12(31):1849-61.

20. Madhusoodanan S, Ting MB, Farah T, Ugur U. Psychiatric aspects of brain tumors: a review. World J Psychiatry. 2015;5(3):273-85. https://doi.org/.549 8/wjp.v5.i3.273

21. Espada Zurera M, Martínez Villena B, Carrero Fernández A. Manejo de las urgencias y emergencias neurooncológicas. Medicine: Programa de Formación Médica Continuada Acreditado. 2019;12(90):5293-302.

22. Zajączkowska M, Kryszkowski W, Talarowska M, Gałecki P. Psychiatric symptoms as possible brain tumor complications: A case report. Medical Science Technology. 2015;56:73-7.

23. Molina JD, Vega LS, Sanz P. Diagnóstico psiquiátrico. Medicine: Programa de Formación Médica Continuada Acreditado. 2019;12(85):4967-74.

24. Sadock BJ, Sadock VA, Ruiz P. Kaplan \& Sadocks synopsis of psychiatry: behavioral sciences, clinical psychiatry. Philadelphia: Lippincott Williams \& Wilkins; 2015.

25. Carmona-Bayonas A, JiménezFonseca P, Vázquez Olmos C, Vega Villar J. Hyperreligiosity in malignant brain tumors: a case report and accompanying bibliographic review. Neurocase. 2017;23(1):88-95. https:// doi.org/.1080/13554794.2016.1265985 
26. Dutschke LL, Steinau S, Wiest R, Walther S. Brain tumor-associated psychosis and spirituality-a case report. Front Psychiatry. 2017;8:237. https://d oi.org/.3389/fpsyt.2017.00237

27. Gyawali S, Sharma P, Mahapatra A. Meningioma and psychiatric symptoms: an individual patient data analysis (review). Asian J Psychiatr. 2019;42:94-103. https://doi.org/.1016/j .ajp.2019.03.029

28. Mocellin R, Walterfang M, Velakoulis D. Neuropsychiatry of complex visual hallucinations. Aust N Z J Psychiatry. 2006;40(9):742-51. https://doi.org/.10 80/j.1440-1614.2006.01878.x

29. Bernstein CA, Poag M, Rubinstein M, Ahn C, Maloy KF y Ying P. The psychotic patient. En: On call psychiatry. New York: Elsevier; 2019.

30. Lorente-Rovira E, Berríos G, McKenna P, MoroIpola M, Villagrán-Moreno JM. Confabulaciones (I): concepto, clasificación y neuropatología. Actas Esp Psiquiatr [internet]. 2011;39(4):251-9. Disponible en: https ://www.actaspsiquiatria.es/repositorio/ 13/72/ESP/13-72-ESP-251-259-34116 5.pdf

31. López EA, Pueyo AA, Maldonado JG. Personalidad esquizotípica y marcadores cognitivos correlatos cognitivos en la esquizotipia psicome\#trica [tesis doctoral]. Universitat de Barcelona, España; 2006.

32. Lee TS. Transient and spontaneously-remitting complex hallucinations in a patient with melanoma and brain metastases. Psychosomatics 2010 MayJun;51(3):267-70. https://doi.org/.117 6/appi.psy.51.3.267
33. Alegría-Loyola MA, GalnaresOlalde JA, Mercado M. Tumores del sistema nervioso central. Rev Méd Inst Mex Seguro Soc [internet]. 2017;55(3):330-40. Disponible en: https://www.medigraph ic.com/pdfs/imss/im-2017/im173i.pdf

34. Aktas A, Walsh D, Hu B. Cancer symptom clusters: an exploratory analysis of eight statistical techniques. J Pain Symptom Manage. 2014;48(6):1254-66.

35. Quispe D, Herold CJ, Kong L y Schröder J. Neurological soft signs (NSS) and gray matter volume (GMV) in first-episode psychosis: an analysis of NSS motor subscores. Psychiatry Res Neuroimaging. 2020 Jun 30;300:111067. https://doi.org/.1016/j. pscychresns.2020.111067

36. Merrell R. Brain tumors. En Rakel $\mathrm{D}$, editor. Conn's current therapy. New York: Elsevier; 2020.

37. Arciniegas DB, Yudofsky SC, Hales RE. Textbook of neuropsychiatry and clinical neurosciences. Washington, DC: The American Psychiatric Association Publishing; 2018.

38. Sinning M. Clasificación de los tumores cerebrales. Rev Méd Clín Las Condes. 2017;28(3):339-42. https://do i.org/.1016/j.rmclc.2017.05.002

39. Chong PK, Loo AV. Visual epilepsy in glioblastoma multiforme. Med J Malaysia [internet]. 2008;63(5):406-7. Disponible en: http://www.e-mjm.org/2 008/v63n5/Visual_Epilepsy.pdf

40. Dombernowsky TW. [Multiple cerebral cavernous haemangiomas in a woman with visual hallucinations]. Ugeskrift Laeger [internet]. 2014 Dec 15;176(25A):V03130207. Danish. Disponible en: https://ugeskriftet.dk/vi denskab/multiple-cerebrale-kavernoes e-haemangiomer-hos-en-kvinde-medsynshallucinationer 
41. Capampangan DJ, Hoerth MT, Drazkowski JF, Lipinski CA. Olfactory and gustatory hallucinations presenting as partial status epilepticus because of glioblastoma multiforme. Ann Emerg Med. 2010 Oct;56(4):374-7. https://doi.org/.1016/ j.annemergmed.2010.02.020

42. Blanke O, Mohr C. Outof-body experience, heautoscopy, and autoscopic hallucination of neurological origin. Brain Res Brain Res Rev. 2005 Dec 1;50(1):184-99. https://doi.org/.1016/j. brainresrev.2005.05.008

43. Bernal B, Guillen M, Ragheb J, et al. Functional imaging localization of complex organic hallucinations. Neurocase. 2019;25(3-4):91-7. https:// doi.org/.1080/13554794.2019.1611866

44. Lim A, Weir P, O'Brien TJ, Kaye $\mathrm{AH}$. Complex visual hallucinations as a presentation of temporal low-grade glioma. New York: Elsevier; 2011.

45. Yoldi Negrete MC, RamírezBermúdez J, Ruiz-Chow A, AguilarVenegas LC, Crail-Meléndez D, Pérez-Esparza R. Alucinaciones visuales en pacientes con privación sensorial: isíndrome de Charles Bonnet? Salud Ment. 2015;38(3):217-24. http://dx.doi.org/1 0.17711/SM.0185-3325.2015.030

46. Sander R, Guerrero V, Perelló M, Ivanov P. Síndrome de Charles Bonnet: las alucinaciones no psiquiátricas. Rev Esp Geriatr Gerontol. 2017;52(2):106. https://doi. org/.1016/j.regg.2016.06.003

47. Kaloshi G, Alikaj V, Rroji A, Vreto G, Petrela M. Visual and auditory hallucinations revealing cerebellar extraventricular neurocytoma: uncommon presentation for uncommon tumor in uncommon location. Gen Hosp Psychiatry. 2013
Nov-Dec;35(6):680.e1-3. https://doi.o rg/.1016/j.genhosppsych.2013.03.011.

48. Calzón GP, Díez RV, Ullán

LG, Iglesias SS. Protocolo diagnóstico del paciente con alucinaciones. Medicine: Programa de Formación Médica Continuada Acreditado. 2007;9(85):5510-12.

49. Pontes S, Calazans R. Regarding hallucination and reality: the psychosis in ICD-10, DSM-IV-TR, and DSM-V and the psychoanalytic counterpoint. Psicol USP. 2017;28(1). https://doi.org/ 10.1590/0103-656420140101

50. Barragán B, Blanch J, Campíñez M. Trastornos de salud mental. En: Atención primaria: problemas de salud en la consulta de medicina de familia. 8. - ed. Barcelona: Elsevier; 2019.

51. Pierre JM. Hallucinations in nonpsychotic disorders. Harv Rev Psychiatry. 2010 JanFeb;18(1):22-35. https://doi.org/.3109/ 10673220903523706

52. Moreno Gra P, Blanco MC. Trastornos mentales orgánicos (III). En: Tratado de psiquiatría [internet]. p. 203-220. Disponible en: https://psiquia tria.com/tratado/cap_10.pdf

53. Friedman DI, Mejico LJ. Traumatismos, fármacos y toxinas. En: Yanoff M, Duker JS, editores. Oftalmología. 5..$^{\mathrm{a}}$ ed. Barcelona: Elsevier; 2019. p. 991-4.

54. Plana Blanco A, González Touya M. Patología neurológica. En: Zurro M, Pérez C, Badia G, editores. Atención primaria: problemas de salud en la consulta de medicina de familia. 8. - ed. Barcelona: Elsevier; 2019. 